\title{
Induction acceleration of heavy ions in the KEK digital accelerator: Demonstration of a fast-cycling induction synchrotron
}

\author{
K. Takayama, ${ }^{1,2,3}$ T. Yoshimoto, ${ }^{1,3}$ M. Barata, ${ }^{1,4}$ Leo Kwee Wah, ${ }^{1,2}$ Liu Xingguang, ${ }^{1,3}$ \\ T. Iwashita, ${ }^{5}$ S. Harada,${ }^{1,4}$ T. Adachi, ${ }^{1,2}$ T. Arai, ${ }^{1}$ D. Arakawa, ${ }^{1}$ H. Asao, ${ }^{6}$ E. Kadokura, ${ }^{1}$ \\ T. Kawakubo, ${ }^{1}$ H. Nakanishi, ${ }^{1}$ Y. Okada ${ }^{6}$ K. Okamura, ${ }^{1,2}$ K. Okazaki, ${ }^{5}$ A. Takagi, ${ }^{1}$ \\ S. Takano, ${ }^{1,5}$ and M. Wake ${ }^{1}$ \\ ${ }^{1}$ High Energy Accelerator Research Organization (KEK), Accelerator Laboratory, 1-1 Oho, \\ Tsukuba, Ibaraki 305-0801, Japan \\ ${ }^{2}$ The Graduate University for Advanced Studies, Hayama, Miura, Kanagawa 240-0193, Japan \\ ${ }^{3}$ Tokyo Institute of Technology, Nagatsuda, Kanagawa 152-8550, Japan \\ ${ }^{4}$ Tokyo City University, Tamatsutsumi, Setagaya, Tokyo 158-8557, Japan \\ ${ }^{5}$ Nippon Advanced Technology Co., Ltd., Tokaimura, Naka, Ibaraki 319-1112 Japan \\ ${ }^{6}$ NEC Network-Sensor Ltd., Futyu, Tokyo 183-8501 Japan \\ (Received 3 February 2013; published 29 January 2014)
}

\begin{abstract}
A fast-cycling induction synchrotron was demonstrated. Ions with extremely low energies and mass-tocharge ratios $(A / Q)$ in the range from 2 to 10 were injected, captured by barrier voltages, and accelerated to the end of the acceleration cycle of $50 \mathrm{~ms}$ by flat pulse voltages generated by pulse transformers referred to as induction cells. Induction acceleration in a wide dynamic frequency range of $56 \mathrm{kHz}$ to $1 \mathrm{MHz}$ was also demonstrated. This accelerator is expected as the next generation of a heavy ion driver for cancer therapy, where a large scale injector is not required. A wide variety of ions for ion energy implantation experiments needing novel materials will be delivered from this compact circular accelerator.
\end{abstract}

DOI: 10.1103/PhysRevSTAB.17.010101

PACS numbers: 41.75.Ak, 29.20.D-, 52.59.Bi

\section{INTRODUCTION}

The KEK digital accelerator (DA) is a small-scale induction synchrotron that does not require a high-energy injector [1]. The induction synchrotron concept was experimentally demonstrated in 2006 using the KEK 12-GeV proton synchrotron, which is a typical slow-cycling synchrotron [2]. The characteristic features of an induction synchrotron are very clear. Instead of a rf cavity typically seen in rf synchrotrons, an induction cell (IC) is employed as the acceleration device, which is simply a one-to-one pulse transformer energized by a switching power supply (SPS) generating pulse voltages. Two types of ICs are utilized, one of which is used only for acceleration and the other generates barrier voltage pulses for beam confinement in the longitudinal direction. The most important feature of the induction synchrotron is that voltage timing is controlled by a gate signal for solid-state switching elements; this gate signal is generated by an intelligent gate controller. In principle, there is no limitation on the available bandwidth for acceleration. These operational characteristics enable acceleration of ions from extremely low energies to the energy allowed by the maximum magnetic rigidity of a

Published by the American Physical Society under the terms of the Creative Commons Attribution 3.0 License. Further distribution of this work must maintain attribution to the author(s) and the published article's title, journal citation, and DOI. specific accelerator ring. Thus, in principle, the KEK-DA is capable of accelerating any species of ion, regardless of its possible charge state [3].

It is noted that Ref. [1] is a kind of design book of the KEK-DA where key accelerator components are explained and the induction acceleration is simply described by computer simulations assuming an ideal situation. The experiment described in Ref. [2] was just the demonstration of the induction synchrotron using a relativistic proton beam, where a change in the revolution frequency $(600 \mathrm{kHz} \rightarrow 800 \mathrm{kHz})$ was rather small. In addition, pulse density control for the induction acceleration voltage that is one of the highlights in the present paper was not employed through the acceleration cycle because of linear ramping of bending magnet fields in the slow cycling synchrotron.

The KEK-DA is an upgrade of the former KEK $500-\mathrm{MeV}$ booster ring, which was in operation between 1973 and 2006. The main guiding magnets, the vacuum chamber, the extraction kicker and septum magnet system, and some beam diagnostic systems were recycled in the upgrade. Consequently, the accelerator ring suffered from undesired features, such as a finite momentum dispersion function at the position of the acceleration device and a pressure level higher than $10^{-8}$ Torr.

In the KEK DA, schematically shown in Fig. 1, a 5-ms ion beam is created in the permanent magnet electron cyclotron resonance ion source (ECRIS) operated at $9.35 \mathrm{GHz}$ [4]. After this, the beam is chopped by the newly developed Einzel lens chopper into $5 \mu$ s portions 


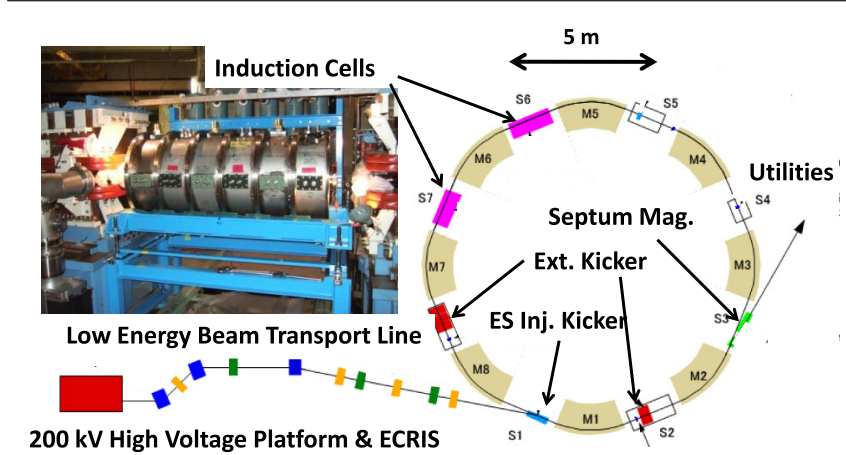

FIG. 1 (color online). Outline of the KEK digital accelerator, where ICs for confinement and acceleration are placed at S6 and S7, respectively. Rectangular shapes on the low-energy beam transport line denote bending, focusing, and defocusing magnets by blue, yellow, and green.

$[5,6]$, subsequently postaccelerated in the acceleration column attached to the $200-\mathrm{kV}$ high-voltage platform, beyond which it propagates through the 15-m low-energy beam transport line to be injected into the ring with the newly developed electrostatic (ES) kicker [7]. The electrostatic kicker voltage is turned off before the injected beam pulse completes a single turn in the DA ring [1], which is a fastcycling synchrotron operated at $10 \mathrm{~Hz}$. The injected beam is captured with a pair of barrier voltage pulses and accelerated with other pulse voltages generated in the ICs. Certain important aspects of the induction acceleration of lowenergy ions with mass-to-charge ratios $(A / Q)$ in the range from 2 to 8 in the KEK-DA are described in this paper, with particular focus on induction acceleration in a wide dynamic frequency range $(82 \mathrm{kHz} \rightarrow 1 \mathrm{MHz})$ in the fast-cycling synchrotron and the intrinsic issues concerning the beam physics associated with acceleration from low energies.

In the future this type of fast cycling induction synchrotron will serve as the next generation of hadron driver for material sciences and medical science, making full use of its advantageous features [1], beyond the conventional electrostatic accelerator, rf linac, rf cyclotron, and rf synchrotron. Here, it is emphasized that the KEK-DA is not only a proofof-principle machine. It is expected to operate as a virtual cosmic ray driver for space science on the ground such as astrobiology [8] and space electronics development [9], and various ion species from $\mathrm{He}$ to $\mathrm{Au}$ in a wide range of energies will be delivered soon after obtaining an official permission of beam delivery. In addition, this accelerator is expected to act as a forerunner to the next generation of digital accelerators developed in the near future [10].

\section{ACCELERATOR RING AND BEAM ORBIT}

As mentioned above, the KEK-DA is a fast-cycling synchrotron operated at $10 \mathrm{~Hz}$. Its lattice consists of eight combined-function magnets. Relevant device and beam parameters are summarized in Table 1, and further details
TABLE I. Device and beam parameters.

Circumference $(C)$ /bending radius $(\rho)$

Injection and extraction magnetic flux density $B_{\min } / B_{\max }$

Betatron tune $\left(\nu_{x} / \nu_{y}\right)$

Transition gamma

Maximum momentum dispersion function

Peak beam intensity

Pulse length

$37.7 \mathrm{~m} / 3.3 \mathrm{~m}$

$0.2-0.5 \mathrm{kG} / 3-8 \mathrm{kG}$

$2.17 / 2.30$

2.25

$1.43 \mathrm{~m}$

$10-100 \mu \mathrm{A}$

$1-5 \mu \mathrm{s}$

are given in Ref. [1]. Guiding magnet fields are ramped in time using a sinusoidal function.

Relatively strong remnant fields of 5-10 G in the guiding magnets are associated with low-energy beam injection, where the relativistic beta is rather small $\left(10^{-2}\right.$ order $)$. This leads to a large closed orbit distortion (COD). Its maximum amplitude of around $20 \mathrm{~mm}$ was monitored. The first and second harmonics were found to be dominant because of $v_{x}$ being close to 2 . The COD was substantially reduced by a newly developed harmonic correction system consisting of figure-8 correction coils wound around each return yolk of the main guiding magnets and independently excited by four power supplies connected to the back-leg coils. Details of the corrected procedure and its result have been discussed elsewhere [11].

\section{GENERATION AND CONTROL OF INDUCTION PULSE VOLTAGE}

The ICs are driven by individual switching power supplies. The timing of the output voltage pulse is uniquely determined by the gate trigger signal for the switching elements employed in the SPS. This gate trigger signal control is the heart of the digital accelerator and consists of a programmable gate control system based on fieldprogrammable gate arrays (FPGAs). A flow chart of the present induction acceleration system control is shown in Fig. 2, where all information required for acceleration is

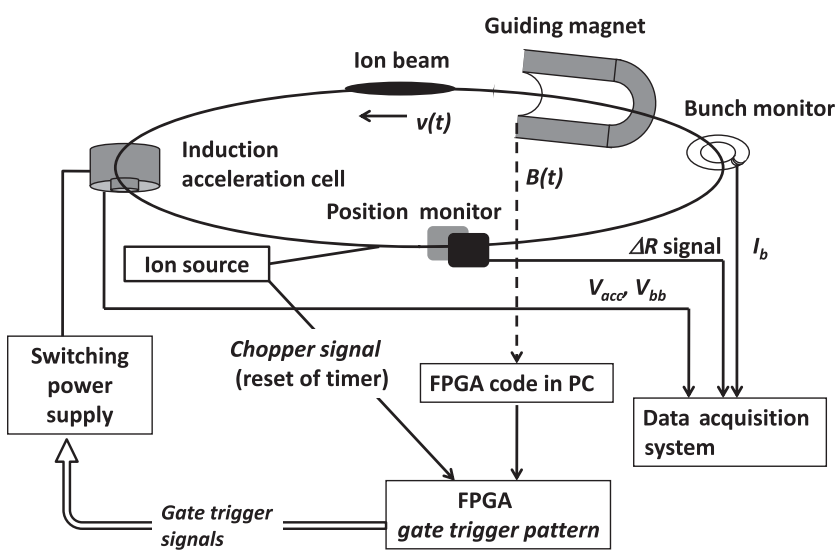

FIG. 2 Flow chart for the induction acceleration system control and data acquisition system. 


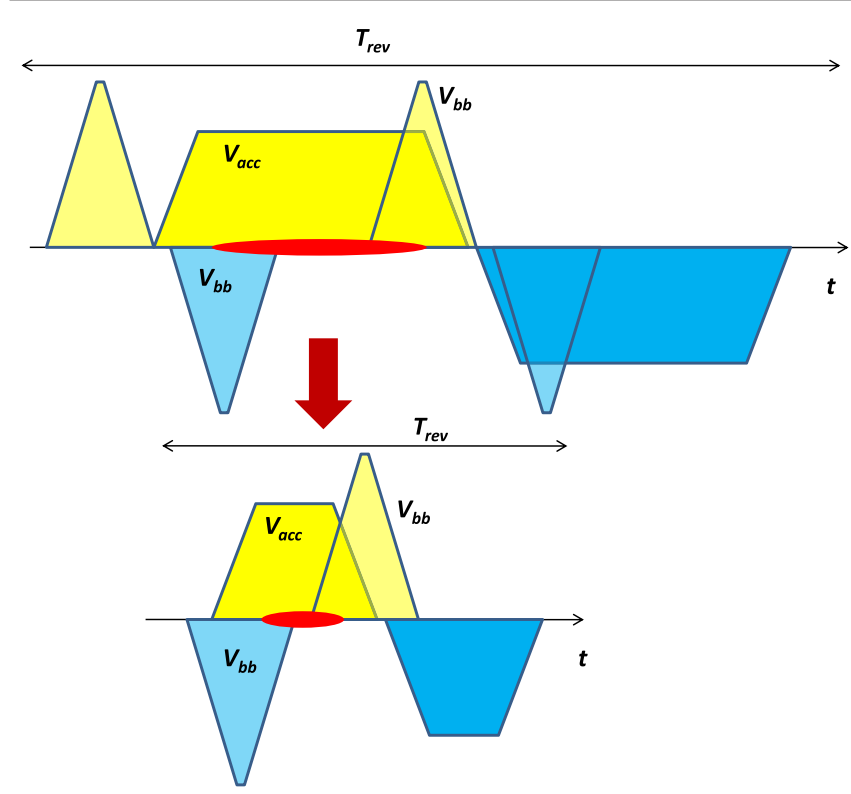

FIG. 3 (color online). Schematic view of capture and acceleration by $V_{\mathrm{bb}}$ and $V_{\mathrm{acc}}$, respectively, at early stage (top) and late stage (bottom) of acceleration.

obtained from the ramping pattern of the guiding magnet and the kinetic balance equation for the centrifugal force in the guiding fields and the Lorentz force, and a complete protocol is created in advance to generate desired gate trigger signals. The chopper signal, which coincides with magnet ramping, governs all subsequent steps in the acceleration cycle. The injected bunch is captured and accelerated by the pulse voltages, as schematically shown in Fig. 3. Meanwhile, details of the data acquisition system and the method of tuning the acceleration parameters are provided in Ref. 12.

\section{BEAM CAPTURE}

The 5- $\mu$ s chopped ion bunch injected into the ring was left to circulate without any longitudinal trapping to simply drift, resulting in a coasting beam within a finite time period depending on the momentum spread. Observations of the bunch structure by utilizing an electrostatic bunch monitor indicated a momentum spread of less than $0.15 \%$, which can be attributed to the fact that ions are accelerated with electrostatic fields upstream.

Next, the bunch was captured by a pair of barrier voltages. Since the beam length is of a few microseconds in the early stage of acceleration and the long time duration between set and reset voltage pulses cannot be taken because of the intrinsic nature of the magnetic material, barrier voltage pulses are generated in sequence by two ICs, as shown in Fig. 3. At a later stage, a single IC provides the necessary barrier voltage pulses. This feature is explained below.

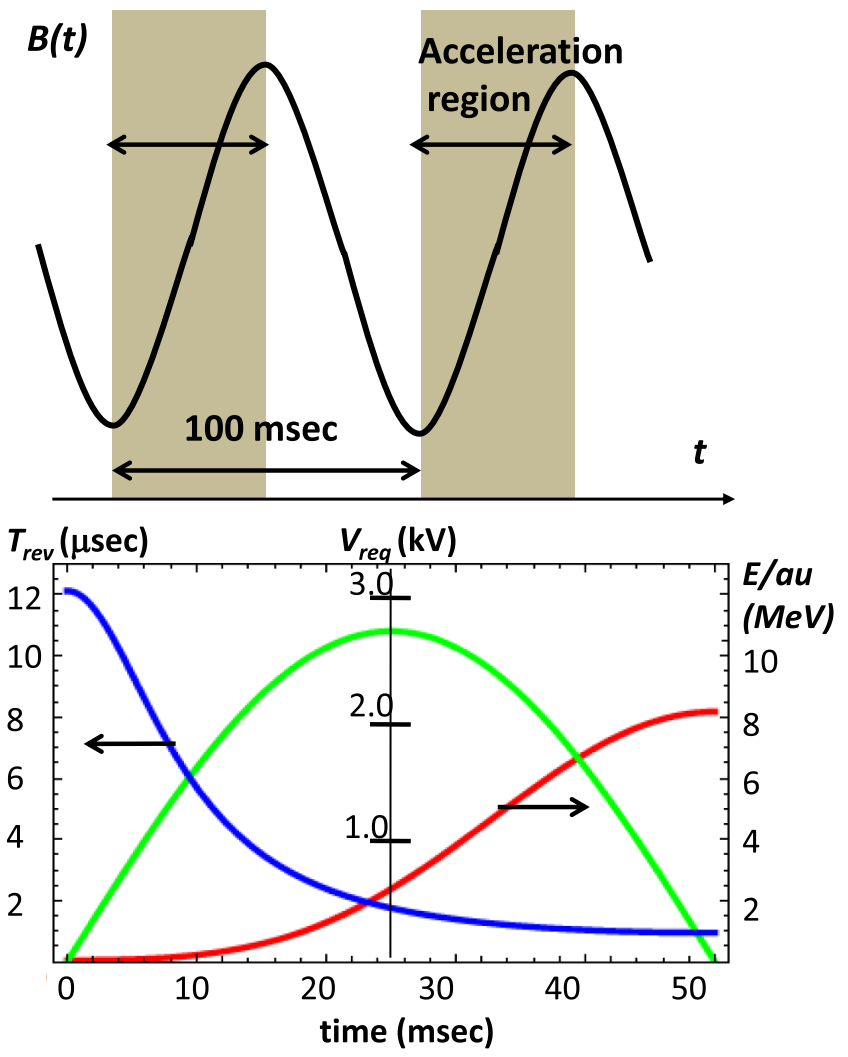

FIG. 4 (color online). Ramping pattern of magnetic flux density, required acceleration voltage per turn $V_{\text {req }}$ (green), revolution period $T_{\text {rev }}$ (blue), and energy per nucleon (red) vs time.

\section{ACCELERATION SCENARIOS}

The present induction acceleration system developed for the induction synchrotron $[1,3]$ has a technical limitation: output voltage height $V_{\text {acc }}$ is fixed to a constant value in the range from $0.3 \mathrm{kV}$ to $2 \mathrm{kV}$ per cell within the acceleration cycle. The acceleration voltage required throughout the acceleration cycle, which is uniquely given in the form of $V_{\text {acc }}=\rho C d B / d t$, is shown in Fig. 4. To meet the requirements of the induction acceleration system, the following scenarios for pulse voltage generation were developed [13] and demonstrated:

(1) Pulse density control.

(2) Superposition in time of pulse voltages generated by different ICs.

In the early and late stages of acceleration, the required acceleration voltage is lower than the output voltage of the IC. Voltage pulse density is controlled such that the gate trigger is generated when the integrated required acceleration voltage reaches $V_{\text {acc }}$ of the IC. Two or three ICs are simultaneously triggered in the time period where the required acceleration voltage $V_{\text {req }}$ exceeds $V_{\text {acc }}$ per cell.

\section{INDUCTION ACCELERATION}

The chopped ion bunch injected at the bottom of the guiding magnetic fields and trapped in the barrier bucket 
TABLE II. Classification of ion acceleration scenarios in KEK-DA and relevant parameters.

\begin{tabular}{lccc}
\hline \hline & $\begin{array}{c}\text { I: Low charge state } \\
\text { corresponding to } \mathrm{Ar}^{+5} \\
\text { and various metal ions }\end{array}$ & $\begin{array}{c}\text { II: Medium charge state } \\
\text { corresponding to } \mathrm{Ar}^{+10}, \\
\mathrm{Ne}^{+5}, \mathrm{O}^{+4}, \mathrm{C}^{+3}, \mathrm{He}^{+1}\end{array}$ & $\begin{array}{c}\text { III: High charge state } \\
\text { corresponding to } \mathrm{Ar}^{+18}, \\
\mathrm{Ne}^{+10}, \mathrm{O}^{+8}, \mathrm{~N}^{+7}, \mathrm{C}^{+6}, \mathrm{He}^{+2}\end{array}$ \\
\hline$A / Q$ & $\sim 8$ & 4 & $\sim 2$ \\
$B_{\min } / B_{\max }(\mathrm{T})$ & $\sim 0.055 / 0.8$ & $0.0389 / 0.5$ & $\sim 0.0274 / 0.25$ \\
Energy $(\mathrm{MeV} / \mathrm{au})$ & 5.25 & 8.2 & $\sim 8.2$ \\
Maximum $V_{\text {req }}(\mathrm{kV})$ & 2.91 & 1.8 & 0.87 \\
$T_{\text {rev }}(\mathrm{inj} / \mathrm{ext})(\mu \mathrm{s})$ & $\sim 17.3 / 1.19$ & $12 / 0.95$ & $\sim 8.6 / 0.95$ \\
\hline \hline
\end{tabular}

was accelerated according to the above acceleration scenario specific to a fast-cycling synchrotron where the operation of multiple ICs with fixed output voltages was combined in a controlled manner. Here, we discuss three typical cases of ion acceleration: (I) a low charge state $(A / Q \geq 8)$, (II) a medium charge state $(A / Q=4)$, and (III) a high charge state $(A / Q \approx 2)$. In the present experiment, the maximum revolution frequency was limited to about $1 \mathrm{MHz}$ because of the limited capabilities of the cooling system for the SPS switching elements and the limitations on the minimum set/reset pulse duration resulting from the finite rising and falling time of the pulse voltages. Consequently, the acceleration parameters listed in Table 2 are realistic. Cases I and II were implemented, and some details regarding case II are given in this paper.

Through the entire acceleration period, the bunch signal $I_{\mathrm{b}}$ was monitored by electrostatic bunch monitors, and $V_{\mathrm{bb}}$ and $V_{\text {acc }}$ were monitored using the current transformers for measuring the currents flowing through the matching resistors connected in parallel to the ICs. The signals of $I_{\mathrm{b}}, V_{\mathrm{bb}}$, and $V_{\mathrm{acc}}$ are plotted as mountain views in time-turn space. Their projections on the time-turn plane clearly represent the evolution of the signals, as shown in Fig. 5. The top figure indicates that the ion bunch captured by $V_{\mathrm{bb}}$ and accelerated by $V_{\mathrm{acc}}$ arrives at the end of the acceleration cycle. In addition, the bunch signal monitored around the end of the acceleration cycle is shown in Fig. 6. Rapid changes in the revolution period are obvious in Fig. 5, where the voltage pulse widths of the constant time period rapidly increase in the phase axis. It is also clear that $V_{\text {acc }}$ pulses are sparse in the early and late stages of acceleration due to the applied pulse density control.

Beam lifetime has been one of our major concerns, even for operation at low current. Significant beam loss was observed toward the final energy level, as shown in Fig. 7. Beam survival at the end of the cycle in the present experiment was about 5\%. Since the cross section for electron capture is inversely proportional to beam velocity as shown in Fig. 8, the lifetime at the low energy region is rather short. Meanwhile, the beam lifetime determined by multiple Coulomb scattering with residual gases is estimated to be around $80-300 \mathrm{~ms}$. Thus, it appears that beam lifetime in the KEK-DA is dominated by electron capture and loss. The change in the number of particles with beam loss through the entire acceleration period is estimated using the following formula:
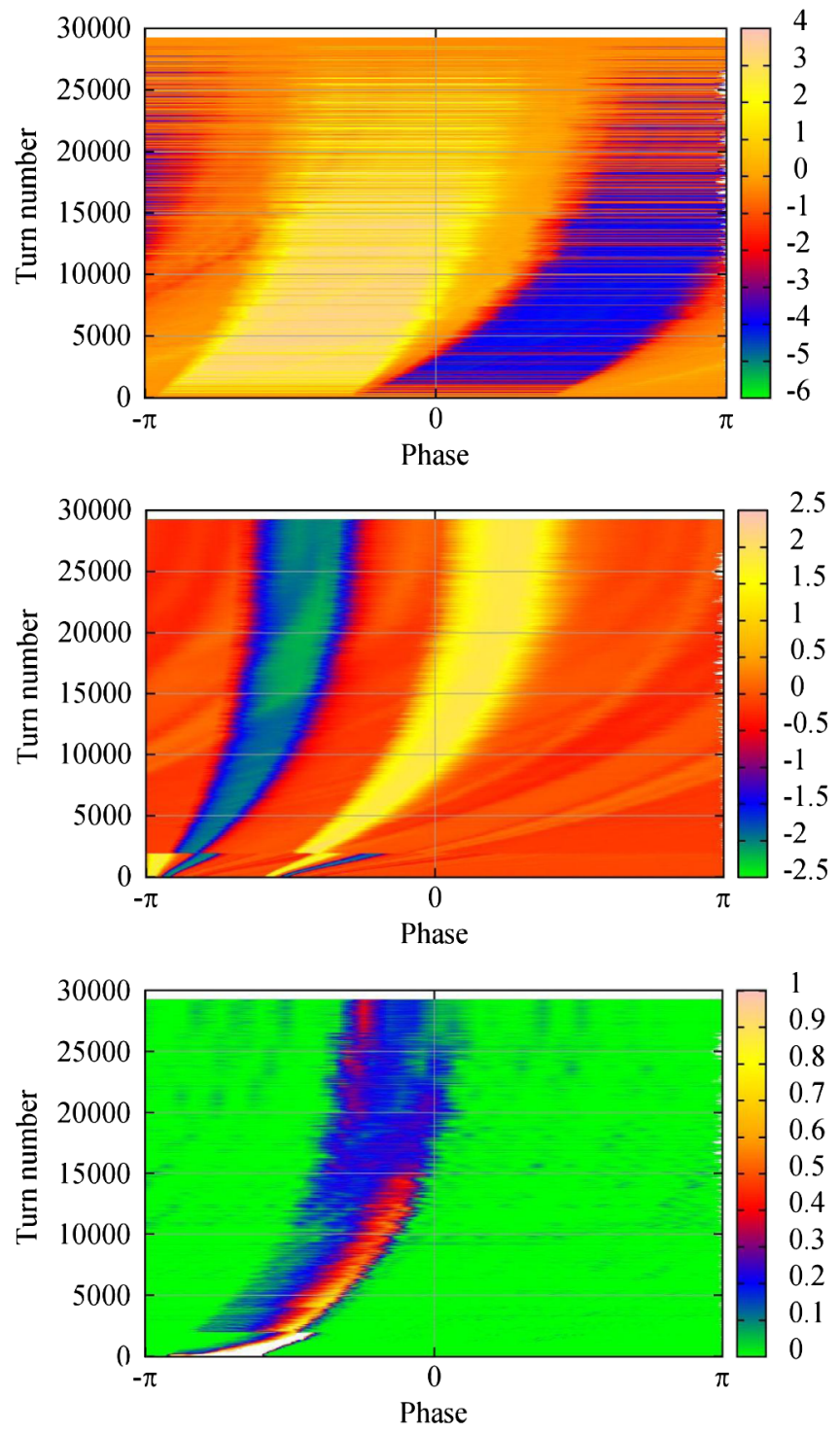

FIG. 5 (color online). For $B_{\max }=0.5$ Tof case II, $V_{\text {acc }}$ (top), $V_{\mathrm{bb}}$ (middle), and bunch signal (bottom), where the horizontal axis is phase and the vertical axis is turn number, in addition yellow and blue in $V_{\mathrm{acc}}$ and $V_{\mathrm{bb}}$ denote set voltages and reset voltages, respectively. 


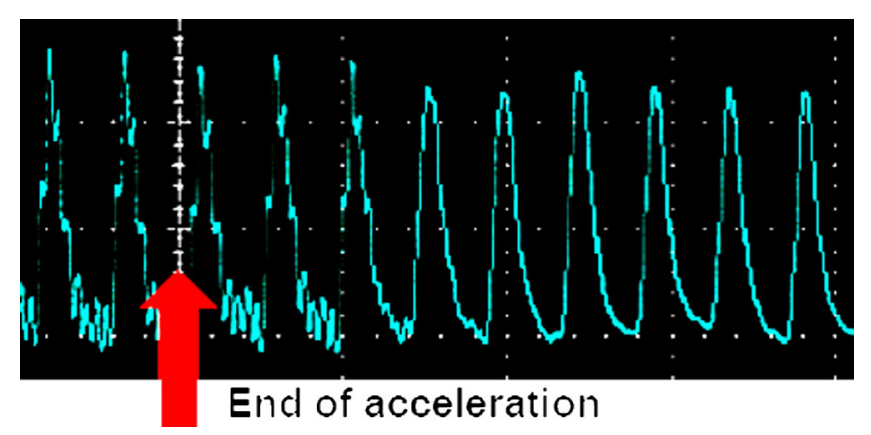

FIG. 6 (color online). Top: reference gate signal for $V_{\mathrm{bb}}$; bottom: bunch signals around the end of acceleration cycle of $50 \mathrm{msec}$.

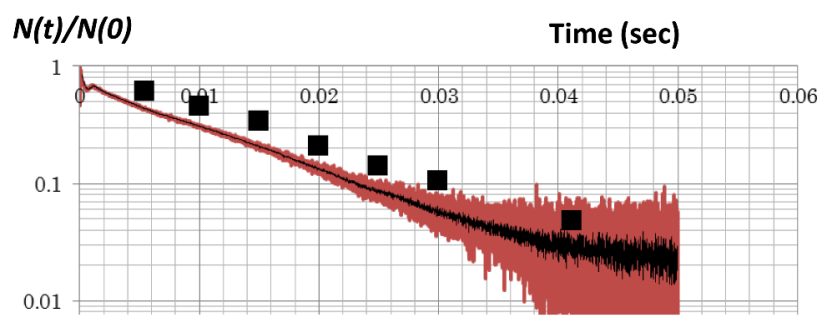

FIG. 7 (color online). Temporal evolution of the number of particles, $N(t) / N(0)$, experiment and calculation (rectangular points).

$$
\frac{n(t)}{n(0)}=\exp \left[-2.12 \times 10^{27} \cdot P(\text { torr }) \cdot \int_{0}^{t}\left(\sigma_{\mathrm{tot}} \beta\right) \cdot d t^{\prime}\right]
$$

The result for $P=2.3 \times 10^{-8}$ Torr is in fairly close agreement with the experimental data until $t=30 \mathrm{~ms}$ after injection. Beyond $40 \mathrm{~ms}$, the linearity of the signal amplifier is not guaranteed because of the small amplitude of the signal.

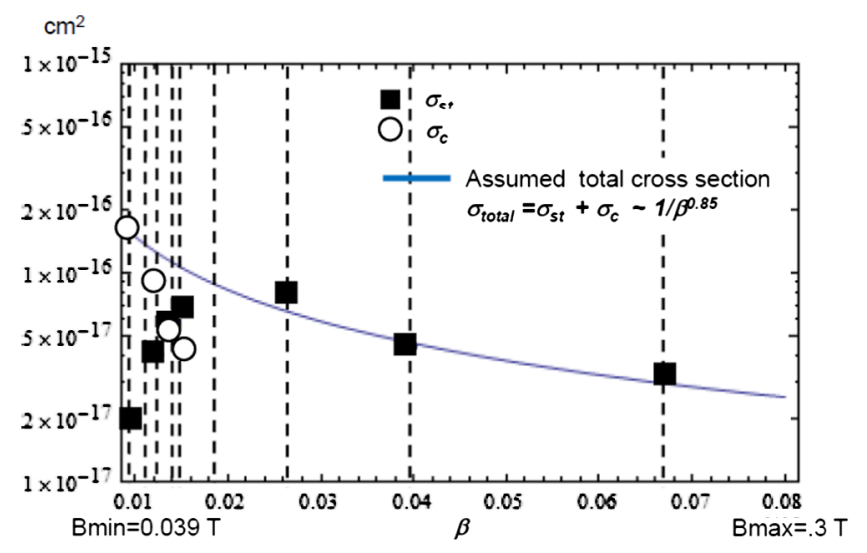

FIG. 8 (color online). Electron capture and loss cross sections of light ion with $A / Q=4$ in Ref. [14] (in $\mathrm{N}_{2}$ ) and Ref. [15] (in air), where $\sigma_{C}$ and $\sigma_{\mathrm{st}}$ are the electron capture and electron stripping cross section, respectively.

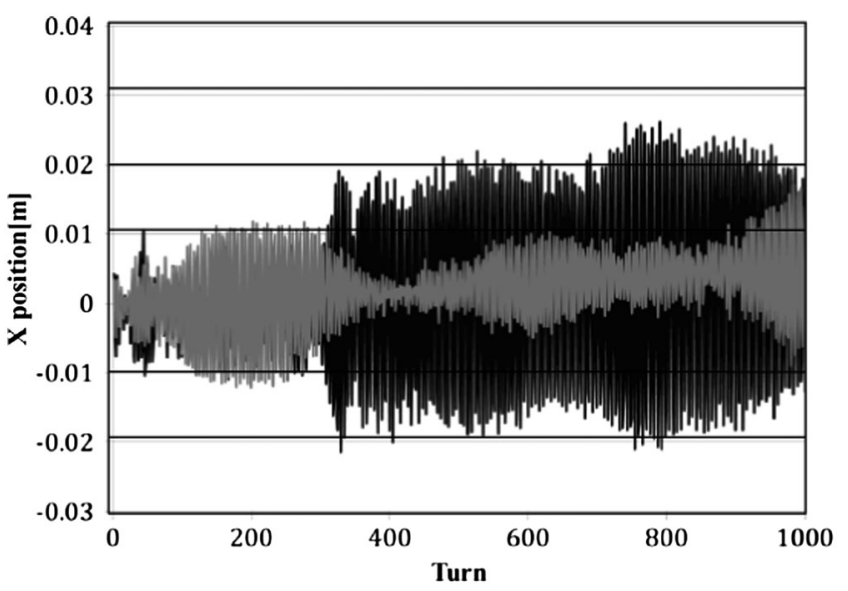

FIG. 9 Temporal evolution of the beam center for plan A (grey) and plan B (black).

\section{EFFECTS OF THE FINITE MOMENTUM DISPERSION FUNCTION}

Note that a finite momentum dispersion function governs particle propagation along the ring, including at the locations of the ICs. This finite momentum dispersion and the discrete acceleration in the early stages under $V_{\text {acc }}$ pulse density control causes a mismatch in the betatron phase space. This mechanism could induce coherent betatron motion an eventually emittance blowup in the horizontal direction, leading to additional beam loss. To confirm this prediction, we tested two voltage plans for $V_{\text {acc }}$ in the early acceleration stage of 1-1000 turns. The bunch monitor signals are compared for the case of fine stepping (denoted as A, $V_{\text {acc }}=0.2 \mathrm{kV}$ for $1-300$ turns, $0.7 \mathrm{kV}$ for 301-800 turns, and $1.2 \mathrm{kV}$ for $>800$ turns), where the pulse density necessarily increases, and the case of constant voltage (denoted as B, $V_{\text {acc }}=1 \mathrm{kV}$ through 1000 turns). The temporal evolution of the beam center for the two cases is shown in Fig. 9, where a relatively large coherent betatron oscillation is induced in case B. Case A occasionally showed substantial beam loss in the early stage of acceleration, when the physical aperture was artificially reduced.

\section{SUMMARY}

Acceleration of heavy ions by pulse voltages discretely generated by ICs was demonstrated in a fast-cycling synchrotron for a wide range of revolution frequencies. We can conclude that the fast-cycling induction synchrotron for acceleration of heavy ions is feasible, although the beam lifetime is substantially limited by the relatively poor vacuum in the KEK-DA. The present results suggest that DA is promising as a means for accelerating heavy ions to high energies without a displacement current $\partial D / \partial t$, which is always required in rf acceleration. The present demonstration will trigger a wide variety of applications of the induction acceleration method far beyond the capability of 
the existing cyclotron and rf synchrotron. For instance, even acceleration of $\mathrm{U} 1+$ begins to be realistic.

\section{ACKNOWLEDGMENTS}

The present study was financially supported by Grants-In-Aid for Scientific Research (S) (KAKENHI No. 20224005) and (A) (KAKENHI No. 23240082).

[1] T. Iwashita, T. Adachi, K. Takayama, T. Arai, Y. Arakida, M. Hashimoto, E. Kadokura, M. Kawai, T. Kwakubo, Tomio Kubo, K. Koyama, H. Nakanishi, K. Okazaki, K. Okamura, H. Someya, A. Takagi, A. Tokuchi, K. W. Leo, and M. Wake, Phys. Rev. ST Accel. Beams 14, 071301 (2011).

[2] K. Takayama, Y. Arakida, T. Dixit, T. Iwashita, T. Kono, E. Nakamura, K. Otsuka, Y. Shimosaki, K. Torikai, and M. Wake, Phys. Rev. Lett. 98, 054801 (2007).

[3] K. Takayama, in Induction Accelerators, edited by K. Takayama and R. Briggs (Springer, New York, 2010), Chap. 11-12, and references therein.

[4] Leo Kwee Wah, K. Takayama, T. Arai, K. Okazaki, A. Takagi, K. Koyama, M. Wake, and Y. Arakida, in Proceedings of Electron Cyclotron Resonance Ion Source, ECRIS-2010 Grenoble, France, 2010 (CLPSC, Grenoble, 2010), p. 150, TUPOT15.

[5] T. Adachi, T. Arai, K. W. Leo, K. Takayama, and A. Tokuchi, Rev. Sci. Instrum. 82, 083305 (2011).

[6] Leo Kwee Wah, T. Adachi, T. Arai, and K. Takayama, Phys. Rev. ST Accel. Beams 16, 043502 (2013).

[7] T. Adachi and T. Kawakubo, Phys. Rev. ST Accel. Beams 16, 053501 (2013).
[8] K. Kobayashi, T. Tonishi, T. Tsuboi, T. Sakai, T. Kaneko, S. Yoshida, T. Takano, and J. Takahashi, Low Temp. Sci., Ser. A 66, 39 (2008) (in Japanese).

[9] K. Takayama, T. Adachi, T. Arai, D. Arakawa, H. Asao, Y. Barata, S. Harada, K. Horioka, T. Iwashita, E. Kadokura, T. Kwakubo, T. Kubo, Kwee Wah Leo, X. Liu, K. Mochiki, N. Munemoto, H. Nakanishi, Y. Okada, K. Okamura, M. Okamura, K. Okazaki, H. Someya, K. Takahashi, S. Takano, M. Wake, and T. Yoshimoto, Nucl. Instrum. Methods Phys. Res., Sect. B 314, 11 (2013).

[10] Conceptual Design Report on Center for Accelerator Research, Phase I and Phase II, Indian Institute of Technology, 2012.

[11] K. Takayama, T. Adachi, T. Arai, D. Arakawa, E. Kadokura, T. Kwakubo, H. Nakanishi, K. Okamura, H. Someya, M. Wake, Kwee Wah Leo, X. Liu, T. Yoshimoto, Y. Barata, S. Harada, H. Asao, Y. Okada, T. Iwashita, Y. Osawa, K. Ise, T. Mizushima, and K. Takagi, Nucl. Instrum. Methods Phys. Res., Sect. A 733, 182 (2014).

[12] T. Yoshimoto, M. Barata, T. Iwashita, S. Harada, D. Arakawa, T. Arai, X. Liu, T. Adachi, H. Asao, E. Kadokura, T. Kawakubo, T. Kubo, K. W. Leo, H. Nakanishi, Y. Okada, K. Okamura, K. Okazaki, H. Someya, K. Takayama, and M. Wake, Nucl. Instrum. Methods Phys. Res., Sect. A 733, 141 (2014).

[13] T. S. Dixit, T. Iwashita, and K. Takayama, Nucl. Instrum. Methods Phys. Res., Sect. A 602, 326 (2009).

[14] I. S. Dmitriev, Ya. A. Teplova, Yu. A. Belkova, N. V. Novikov, and Yu. A. Fainberg, At. Data Nucl. Data Tables 96, 85 (2010).

[15] S. K. Allison, J. Cuevas, and P. G. Murphy, Phys. Rev. 102, 1041 (1956). 